\title{
Comparative analysis ofthe functioning of sieve modulesforgrain cleaning machines
}

\author{
Andrey Butovchenko ${ }^{1, *}$, Artyom Doroshenko ${ }^{1}$, Aleksandr Kol'cov $^{1}$, and Valentina Serdyuk ${ }^{1}$ \\ ${ }^{1}$ Don State Technical University, 344000 Rostov-on-Don, Russian Federation
}

\begin{abstract}
Thearticlegives the calculationdiagrams of the sieve modules for grain cleaning machines whichallow to analyze the operation of the grain cleaning machines, and the trajectory of materials moving within the machine.A generalized mathematical model of the functioning of air-sieve machines and sieve modules is presented. Based on the regression equations adequately describing the processes occurring on the sieves, the simulation of the calculation schemes of the sieve modules of grain cleaning machineswas carried out.The modeling results, such as the purity of the grain material, the completeness of the separation of impurities, the content of weed and grain impurities are presented in the form of dependencies, allowing them to be analyzed and selected the most efficient grain cleaning machine for the production line of seed material.
\end{abstract}

\section{Introduction}

The trend forecast for grain production and consumption, according to the Ministry of Agriculture of the Russian Federationshows stable growth due to growth of demand both among consumers andprocessors. The implementation of this scenario is possible due to increased productivity, which is not possible without the use of high-quality seeds. Existing requirements for seed are regulated according to accordance with GOST R 52325-2005.To meet the requirements for the purity of the seed material after combine harvesting, a postharvest operation is carried out, as a result of which weed and grain impurities from the harvested seed material, bringing the purity of the seed to $99 \%$.

\section{Materials and Methods}

Cleaning takes place on grain cleaning units, representing a production line, which includes a set of machines that allow to separate impurities different from the main crop in terms of aerodynamic properties, dimensional characteristics, roughness, specific weight.The main machines that have a significant impact on the efficiency of the post-harvest process are airsieves cleaning machines. Currently, there is a wide variety of these machines, differing in the structure of the sieve modules, the sizes of the sieve included in them, the kinematic modes, the cleaning and drive system of the sieve, the presence of one or two air separators, types of air separators and other signs affecting the efficiency of the separation process in

${ }^{*}$ Corresponding author: AVButovchenko@yandex.ru 
these machines. When creating new seed cleaning units and modernizing old ones, it becomes necessary to select the optimal machine that meets the conditions of the production line.To do this, it is necessary to conduct a comparative analysis of the functioning of the sieve modules for existing grain cleaning machines [1-7].

At the first stage, a market analysis was carried out, a search for air-sieve grain cleaning machines, an analysis of the functioning of their sieve modules, the schemes of which are presented in Figure 1.

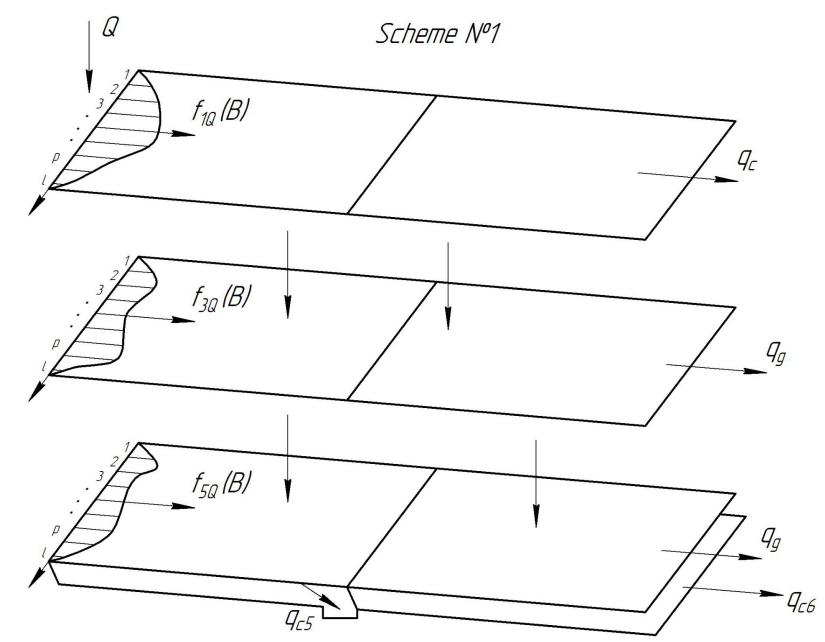

a)

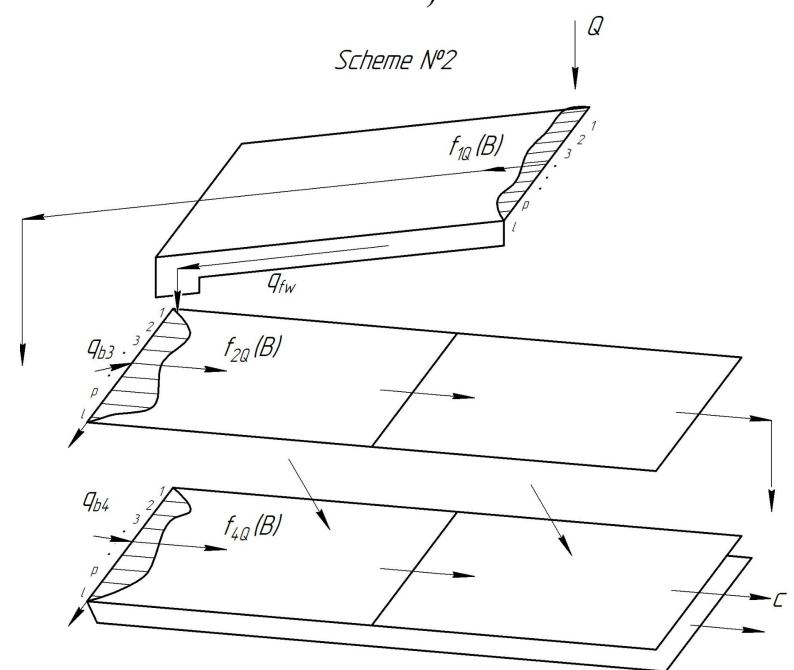

b) 


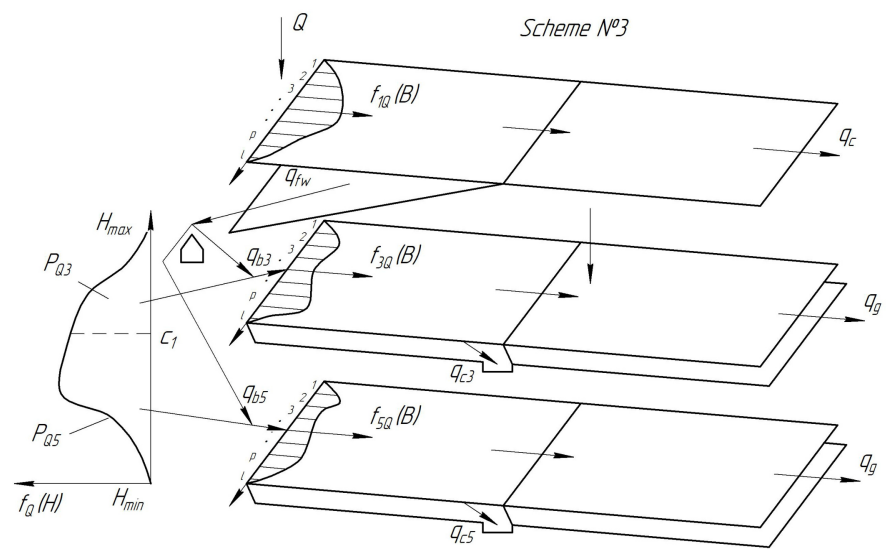

c)

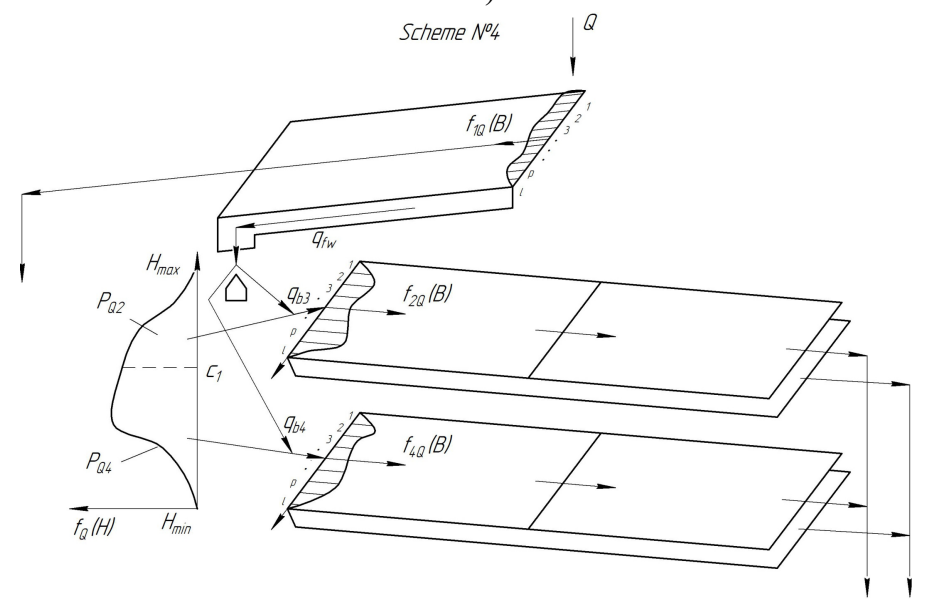

d)

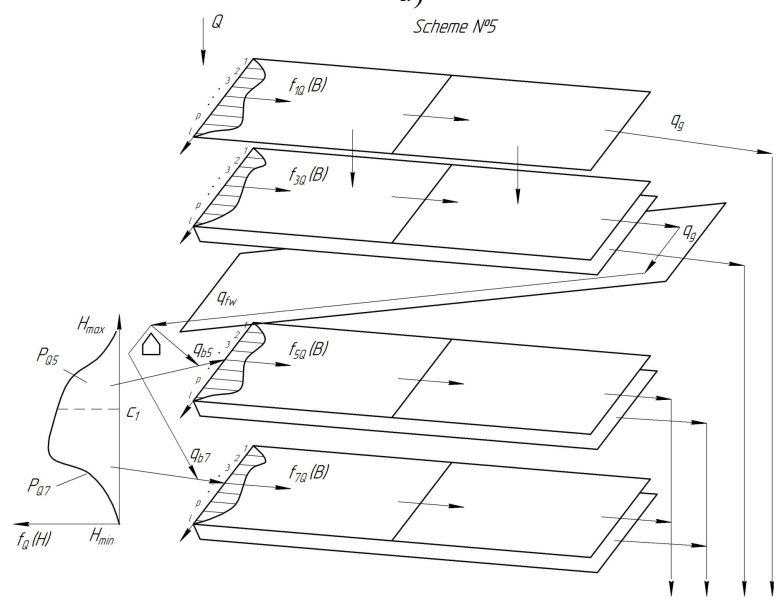

e) 


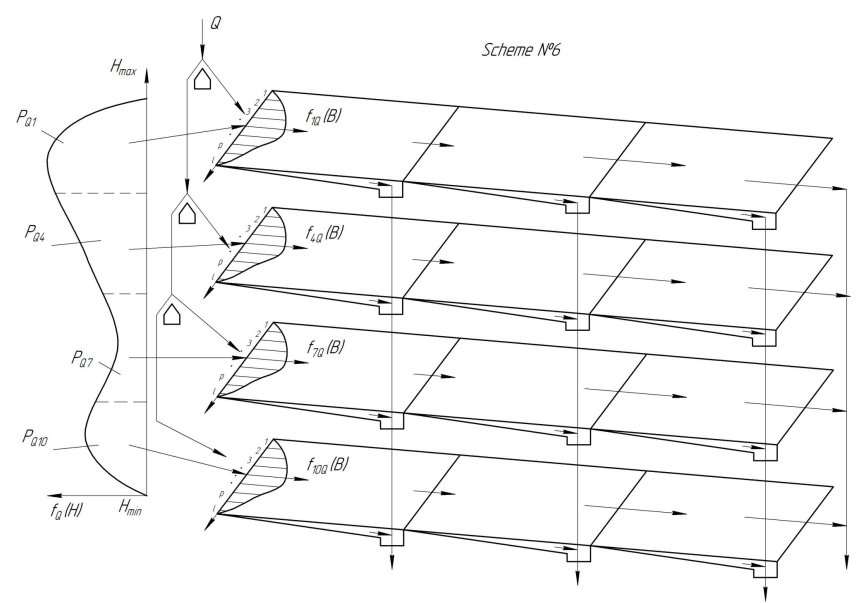

f)

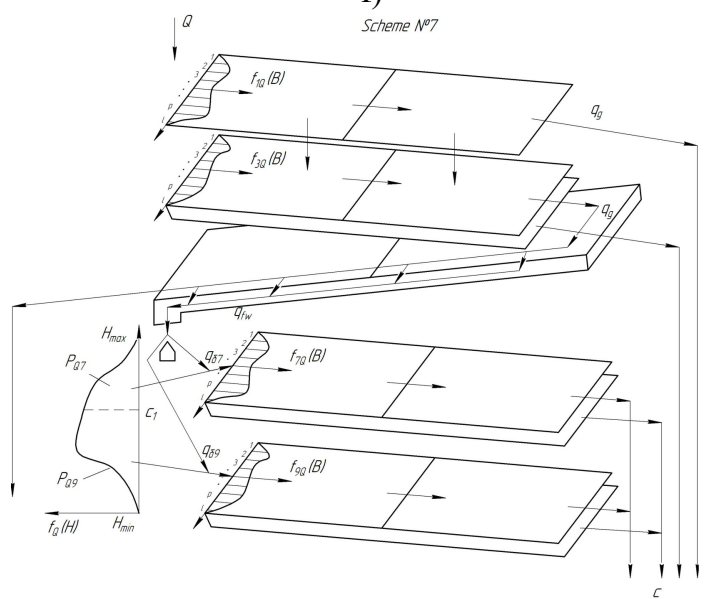

g)

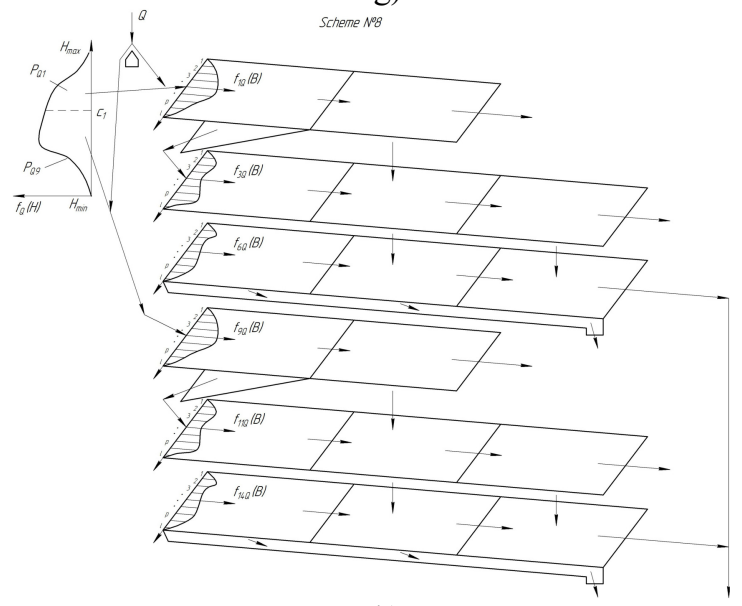

h) 


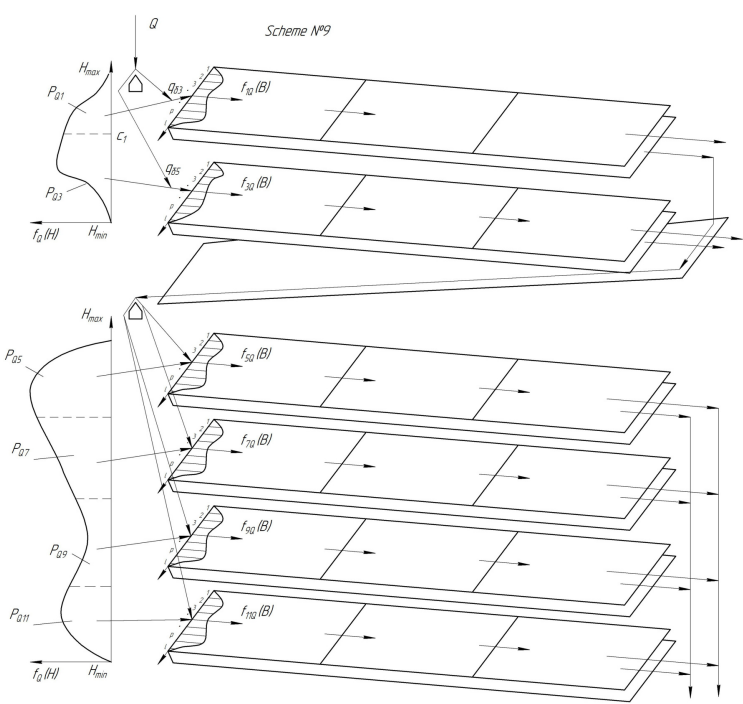

i)

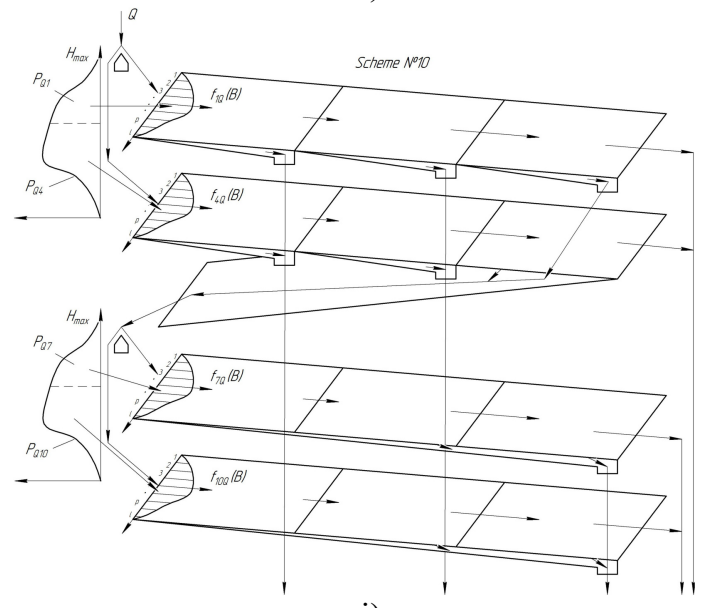

j)

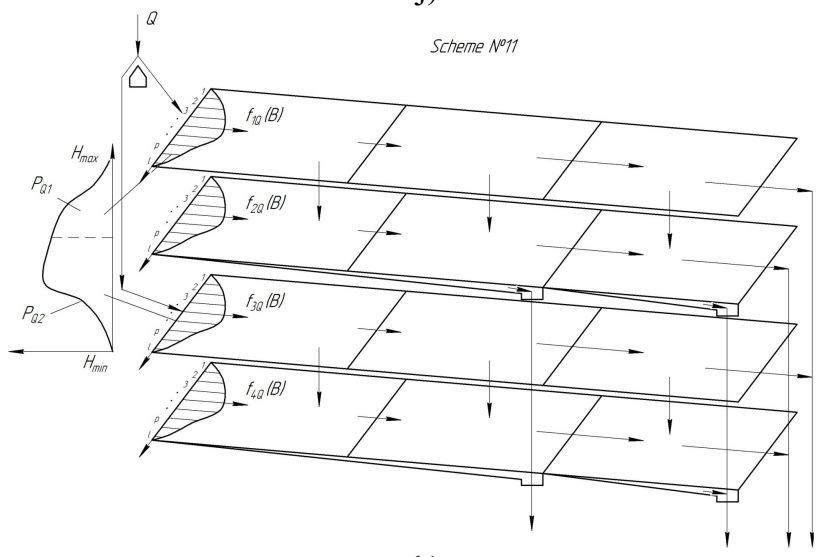

$\mathrm{k})$ 


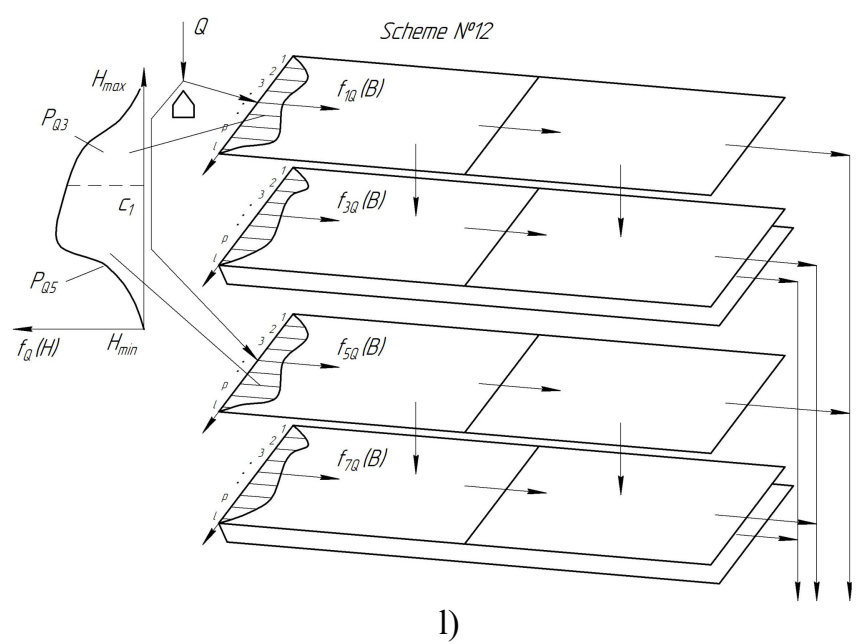

Fig. 1.Calculation schemes of sieve modules of type:(a) -Petkus A12;(b,d) - Clipper Prelude,SGM 25/10/5;(c) - Petkus A09;(e)- Petkus U12, Petkus U15;(f)- ASM-6, SGC-50/25/10HSU-60;(g) Cimbria;(h) -Petkus V12,Petkus V15;(i) - Petkus M12, Petkus M15;(j) -Prototype;(k) - GFC80/40/20;(1) GASS-20A, MPU-70, VSC-50.

At this time, there are new methods that allow to carry out a full-fledged simulation of the operation of existing machines[8-9], however, at present, there is nodescription for the properties of various seeds material and all the impurities included in, as it necessary for the statement of the problem, in addition,the activedevelopment of these techniques, in relation to solution of this problem, is hampered by the need for very large computing power. Therefore, the previously created mathematical model of the functioning of the air-sieve grain cleaning machine is used [10-11].

A mathematical model for the process of functioning of an air-sieve grain cleaning machine, as a closed quasi-static system with a given $\mathrm{K}_{\delta M}$ functional scheme, can be written in general form as follow:

$$
\begin{aligned}
\boldsymbol{E}_{\boldsymbol{f} \boldsymbol{M} \boldsymbol{M}} & =\left\{\boldsymbol{F}, \boldsymbol{A}, \boldsymbol{G}_{\boldsymbol{\delta}}\left[\boldsymbol{k}_{\boldsymbol{\delta} \boldsymbol{M}}(\boldsymbol{x}), \mathrm{T}_{\boldsymbol{\delta} \boldsymbol{M}}(\mathrm{x})\right]\right\} \rightarrow \boldsymbol{m a x}, \\
\boldsymbol{A}_{\boldsymbol{M}} & \subset \overrightarrow{\mathrm{A}}_{\mathrm{M}}, \quad \boldsymbol{F} \subset \overrightarrow{\mathrm{F}}, \quad \boldsymbol{x} \in \boldsymbol{G}_{\boldsymbol{M}}(\boldsymbol{x}, \boldsymbol{u}) .
\end{aligned}
$$

limitations:

$$
\boldsymbol{b}_{w} \leq\left[\boldsymbol{b}_{w}\right], \boldsymbol{b}_{g i} \leq\left[\boldsymbol{b}_{g i}\right], \boldsymbol{\delta}_{p} \leq\left[\boldsymbol{\delta}_{p}\right]
$$

Here: $\overrightarrow{\mathbf{F}}$-is the vector of input influences on the operation system adopted in the grain cleaning machine

$$
\overrightarrow{\mathrm{F}}=\left\{\boldsymbol{Q}, \boldsymbol{a}_{\boldsymbol{j}}, \boldsymbol{W}, \boldsymbol{M}\left(\boldsymbol{b}_{j}\right), \boldsymbol{\sigma}\left(\boldsymbol{b}_{j}\right), \boldsymbol{f}_{\boldsymbol{Q}}(\boldsymbol{B}), \boldsymbol{f}_{\boldsymbol{Q}}(\boldsymbol{H})\right\}
$$

Where: $Q$ - is supply of grain material to a grain-cleaning machine $\mathrm{a}_{j}, W$ - the content of $j$-thecomponents in the source material, their moisture content; $M\left(b_{j}\right), \sigma^{2}\left(b_{j}\right)$ - mathematical expectations and variances of sizes of signs of separation of $j$-th components; $b_{w} ., b_{g i}, \delta_{g l}$-are the content of purified grain of weed, grain impurities and grain loss.

$\vec{A}$ - vectorof control factors of the system ensuring its functioning. 


$$
\overrightarrow{\mathrm{A}}=\left\{\boldsymbol{B}_{P C i}, \boldsymbol{h}_{P C i}, \boldsymbol{V}_{\boldsymbol{b i}}, \boldsymbol{f}_{\boldsymbol{q}}\left(\boldsymbol{B}_{P C}\right), \boldsymbol{f}_{\boldsymbol{v}}\left(\mathrm{B}_{P C}\right), \boldsymbol{P}_{\boldsymbol{f d}}\left(\boldsymbol{P}_{\boldsymbol{i}}\right), \boldsymbol{f}_{\boldsymbol{Q}}(\boldsymbol{H}), \boldsymbol{f}_{\boldsymbol{Q}}(\boldsymbol{B}), \mathrm{K}_{\delta \boldsymbol{M}}(\mathrm{x})\right\}
$$

Where: $B_{P C i}, h_{P C i}$ - are the width and depth of the $i$-th in pneumatic separator system; $V_{b i}$ - the working speed of the airflow in the $i$-thair separator; probability distribution density $f_{q}\left(B_{n}\right)$ - the grain material and air flow $f_{v}\left(B_{P C}\right)$ in $\operatorname{width} B_{P C}$; air separatorP $P_{f d}\left(P_{i}\right)$ functionalschemes of sieve modules;probability density distribution of the grain material in the sieve tiers $f_{O_{x}}(H)$ and the width of the sieves $f_{Q x}(B)$ in sieve modules of grain cleaning machines; $\mathrm{K}_{\delta M}(\mathrm{x})$-functional diagram and parameters of the air sieve cleaning machine.

Asx- is a set of technological operations from $G_{\mathrm{M}}(x, u)$ sets implemented in $K_{\delta \mathrm{M}}$ the functional diagram of the air sieve cleaning machine. $G_{\delta}\left[\mathrm{K}_{\delta M}(\mathrm{x}), T_{\delta}(\mathrm{x})\right]$ - the functional that determines the indicators of the technological process in the air sieve cleaning machine(completeness of separation of $j$-th components, grain loss, the content of the purified grain of other $\mathrm{j}_{\text {-th }}$ components),for adopted $\mathrm{K}_{\delta M}(\mathrm{x})$ in scheme (Figure 1).

The output performance of the grain cleaning machine is determined by the vector $\overrightarrow{\mathrm{B}}_{M}$, independent arguments of which are random in a probabilistic-statistical sense.

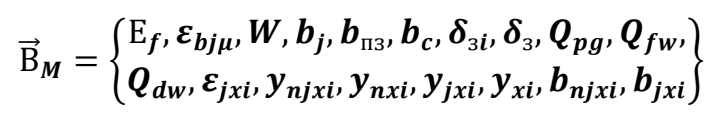

Where: $\mathrm{E}_{f}-$ The criterion of efficiency of realization for the air sieve cleaning machine technological process; $\varepsilon_{b j \mu}$ - completeness of the separation material of $\mathrm{j}_{\text {-th }}$ components from the grain material, $\delta_{g l i}, \delta_{g l}$ - grain losses on $x i$ operations and general; $Q_{p g}, Q_{f w}, Q_{d w}$ - the mass yield of cleaned grain, feed waste and waste of different categories; weight $y_{n j x i}, y_{n x i} j$ thand all components of the grain material after implementation $x i$ operation. weight $y_{j x i}, y_{x i}$ $j$-th and all components isolated from the grain material after implementation $x$ ioperation; the content of the $j$-th component in the mass $b_{n j x i}$ cleaned material and in allocated fractions $b_{j x i}$; the completeness of the passage $\varepsilon_{j x i} j$-th the component in the cleaned grain material after the $x i$ operation.

The input effect on the lattice module under consideration is expressed by a vector $\vec{F}_{p}$, the independent arguments of which are random, in the probability-statistical sense of the magnitude.

$$
\vec{F}_{p}=\left[Q_{p}, a_{p j}, W, M\left(b_{j}\right), \sigma^{2}\left(b_{j}\right), f_{Q}\left(B_{i}\right), f_{Q}(H), f\left(l_{i}\right)\right]
$$

The active means determining the efficiency of the lattice module was determined by the vector $\overrightarrow{\mathrm{A}}_{\mathrm{p}}$

$$
\overrightarrow{\mathrm{A}}_{\mathrm{p}}=\left[\alpha_{p i}, \beta_{i}, R_{i}, n_{i}, T_{i}, l_{i}, B_{i}, b_{i}, c, K_{\mu}(x)\right]
$$

Where: $Q_{p}, a_{p j}, W$-the supply of grain material, its moisture content $\mathrm{W}$, content of $j$-th components in it; $M\left(b_{j}\right), \sigma^{2}\left(b_{j}\right)$ - statistical characteristics of the size of the $j$-thcomponent separation feature; $f_{Q}\left(B_{i}\right), f_{Q}(H)$-the probability density of the distribution of the $j$-th component across the width $B_{i} i$-th sieve in the tier and the height of the tiers in the sieve module; $f\left(l_{i}\right)$ - the regularity of grain material entering the sieves; $\alpha_{p i}, \beta_{i}, R_{i}, n_{i}$ inclination angles of the $i$-th sieves to the horizon, directivity, amplitude, and frequency of sieves oscillations; $T_{i}$ - type of the $i$-th sieve; $l_{i}, B_{i}$ - the length and width of the sieves in the module; $b_{i}$ - the working size of $i$-th sieve holes; $c$-the number of sieves in the sieve module; $K_{\mu}(x)$ - functional diagram of the sieve module. 
Consider in the general case a multi-tiered lattice module with a parallel arrangement of lattice tiers (Figure 2$)$ with heterogeneous in length $\left(l_{11}, \ldots l_{1 n}\right)$ and height $(1,2, \ldots, \mathrm{K})$ in General, the tiers of sieves receive $q_{1 j}, q_{2 j}, \ldots, q_{n j}, q_{\kappa} j$ and quantity of $j_{\text {-th }}$ component of bulk material from their ends, determined by the probability density function of the distribution $f_{Q}(H)$ of a random variable $Q$ (supply of bulk material to tiers $(1,2, \ldots, 4$ sieves) over the height $H$ of the tier arrangement of the sieves, and the distribution of the random variable $q_{\delta}$ along the width $\mathrm{B}_{\mathrm{p}}$ of separators in tiers is determined by the probability density $f_{n q}(B)$. As an assumption, here and further we consider that the flow of bulk materials on the tiers of the sieves is constant in time (process quasi-static) and with a uniform distribution of the $j$-th components in the bulk material $(j=1,2, \ldots)$. Accepting the well-known hypothesis about the additivity of the process of separation of bulk material on the $p_{\text {-th }}$ sections of the sieve tiers, the completeness of sifting of the $j$-th component on the $\delta$-th sieve tier is determined by the well-known expression [10].

$$
\begin{gathered}
\varepsilon_{\delta j}=\frac{\sum_{p=1}^{e} q_{\delta p} \cdot a_{j} \cdot \varepsilon_{\delta j}\left(q_{\delta p}\right)}{q_{\delta} \cdot a_{j} \cdot e} \\
q_{\delta p}=\frac{B \cdot Q}{\mathrm{\kappa}} P_{\delta Q p}
\end{gathered}
$$

Where: $q_{\delta p}$ - reduced flow of bulk material to the $p_{\text {-th }}$ section (width $\left.B\right)(p=1,2, \ldots, e)$ of the $n$-th lattice tier; $\mathrm{k}=\mathrm{B} \backslash \mathrm{e}$ is the width of the $p$-th section of the sieve tier; $P_{\delta Q p}$ - the probability of a random variable $Q$ falling into the $p$-th section along the width of the $\delta$-th lattice layer.

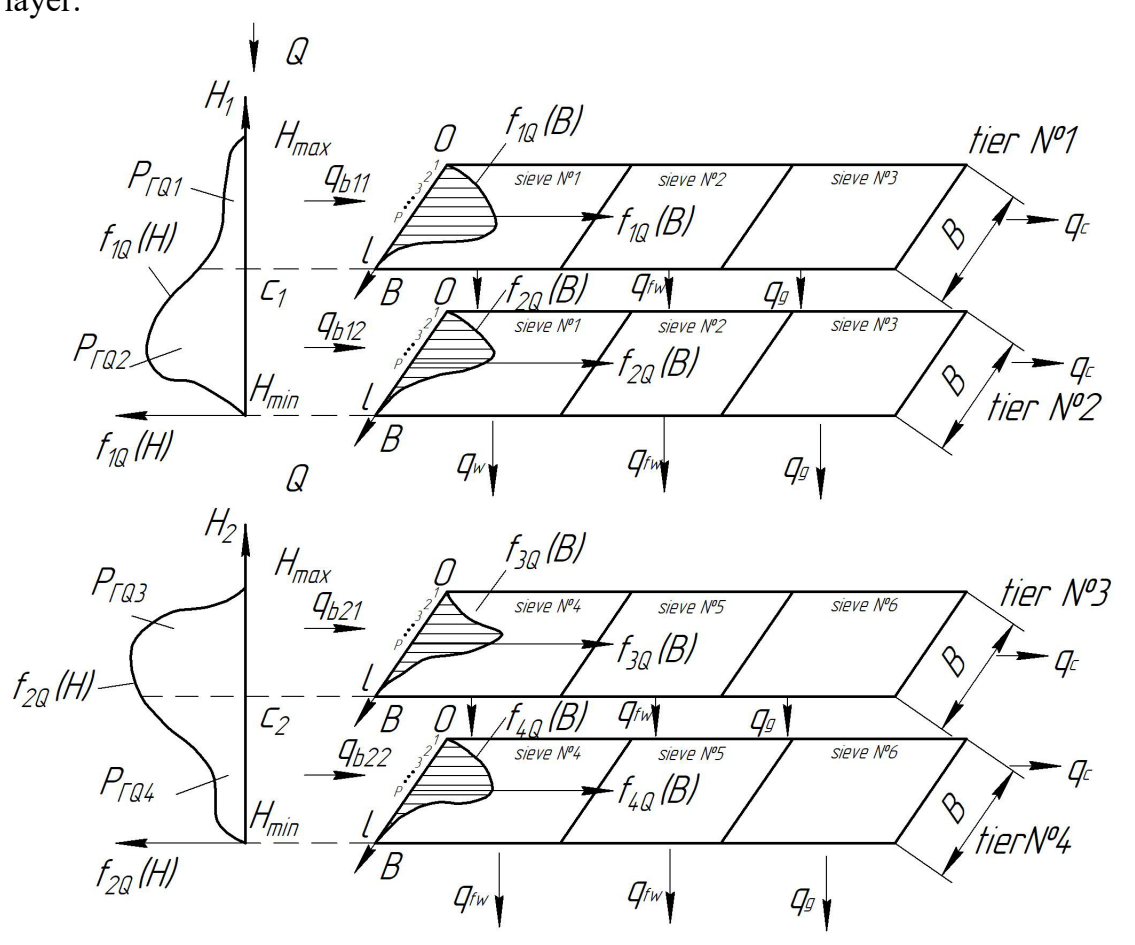

Fig. 2. The probability density $f_{1 Q}(H)$ and $f_{2 Q}(H)$ the grain material supply to the first and second sieve modules and the probability density $f_{i Q}(B)$ of the distribution of grain material across the width of the ith tiers sieves $(i=1,2, \ldots, 4) ;\left(\mathrm{c}-\mathrm{H}_{\min }\right)$ - the distance between the tiers of sieves 
In this case, the supply $q_{\delta}$ of bulk material to $\delta_{\text {-th }}$ sieve tier

$$
q_{\delta}=Q \cdot P_{\Gamma Q} \cdot \kappa
$$

Where: $\varepsilon_{\delta j}, q_{\delta p}$-the sieving completeness of the $\mathrm{j}_{\text {-th }}$ component on the $p$-th section of the $\delta_{\text {-th }}$ lattice layer when the $q_{\delta p}$ bulk material is fed to this section, will be determined from various expressions [10] depending on many factors.

$$
\varepsilon_{\delta j}\left(q_{\delta p}\right)=f\left(Q, a_{j}, W, \gamma, f_{j}, L_{\delta m}, d_{m}, \alpha_{m}, \beta_{m}, R_{m}, N_{m}, n\right)
$$

Where: $f_{j}, j_{j} \cdot \gamma$ - type of bulk material (grain, grass seeds, etc.) and its density; $L_{\delta m}-$ length of the $m_{\text {-th }}$ sieve of the $\delta_{\text {-th }}$ lattice tier; $d_{\delta m}$ - the working size of the holes of the $m$-th sieve of the $\delta_{\text {-th }}$ tier; $\alpha_{\delta m}, \beta_{\delta m}, R_{\delta m}, N_{\delta m}$ - the angle of inclination to the horizon, directivity, amplitude and frequency of oscillations of the $m$-th sieve in the $\delta$-th tier; $n$ - number of sieves per tier.

Obviously, for the known $f_{Q}(H)$ and $f_{q}(B)$ the sifting completeness of the $j$-th component through the $\delta_{\text {-th }}$ lattice tier [11].

$$
\varepsilon_{\delta j}=\frac{\sum_{p=1}^{l} Q \cdot \frac{\int_{r-1}^{r} f_{Q}(H) d H}{\int_{r=1}^{c} f_{Q}(H) d H} \cdot \frac{\int_{p-1}^{p} f_{\delta q^{(B) d B}}}{\int_{p=1}^{c} f_{\delta q}(B) d B} \cdot a_{j \delta} \cdot \varepsilon_{\delta j p}\left(q_{\delta p}\right)}{\sum_{j} q_{\delta} \cdot a_{j}}
$$

\section{Results}

To simulate the processes of separation of grain material on various structures of sieve modules, well-known mathematical models were used [11], the adequacy of which was proved earlier.

The following conditions were accepted for modeling:the probability density $f_{Q}(B)$ of the grain material feed distribution over the width $B$ of the sieve in the tiers corresponds to the normal law; $W$ - the humidity of the heterogeneous granular medium (wheat grain) was $14 \% ; L=790 \mathrm{~mm}$ - the length of the sieve included in the sieve module; $\alpha=6^{\circ}$ - the angle of inclination of the sieves to the horizon; $\beta=0$ direction of oscillations; $n=420 \mathrm{~min}-1$ oscillation frequency; $R=8 \mathrm{~mm}$, the amplitude of the oscillations of the sieves; $B=990 \mathrm{~mm}$ - sieve width; sieves are flat, with the dimensions of rectangular holes $1.7 \times 20 \mathrm{~mm}, 2.0 \times 20 \mathrm{~mm}, 2.2 \times 20 \mathrm{~mm}$, $2.6 \times 20 \mathrm{~mm}, 3.0 \times 20 \mathrm{~mm}, 3.6 \times 20 \mathrm{~mm}$.

The composition of the initial purified heterogeneous granular medium (wheat grain) for the simulation is assumed constant: $a_{1}=93.66 \%$ - wheat grain content; $a_{2}=0.15 \%$ - the content of straw impurities; $a_{3}=0.51 \%$ - the content of large impurities; $a_{4}=4.25 \%$ - the content of thin grain; $a_{5}=0.31 \%$ - the content of small weed impurities; $a_{6}=1 \%$ is the content of crushed grain; $a_{7}=0,12 \%$ - the content of weed seeds; $M\left(b_{i}\right)=2.7465 \mathrm{~mm}$-average grain thickness; $\sigma^{2}\left(b_{j}\right)=0,042 \mathrm{~mm}^{2}-$ the dispersion of the thickness of the grain; $\mathrm{a}_{g p} \geq\left[\mathrm{a}_{g p}\right],\left[\mathrm{a}_{g p}\right]=$ $99,5 \%$ - set purity of refined grain.

The separation criterion $E_{f} \rightarrow$ maxwas used as the main criterion [11]. Limitations:grain loss $\delta_{g l} \leq 0,5 \%$; grain yield in feed waste $\delta_{f w} \leq 3 \%$. The simulation results are shown in Figures 3-6. 


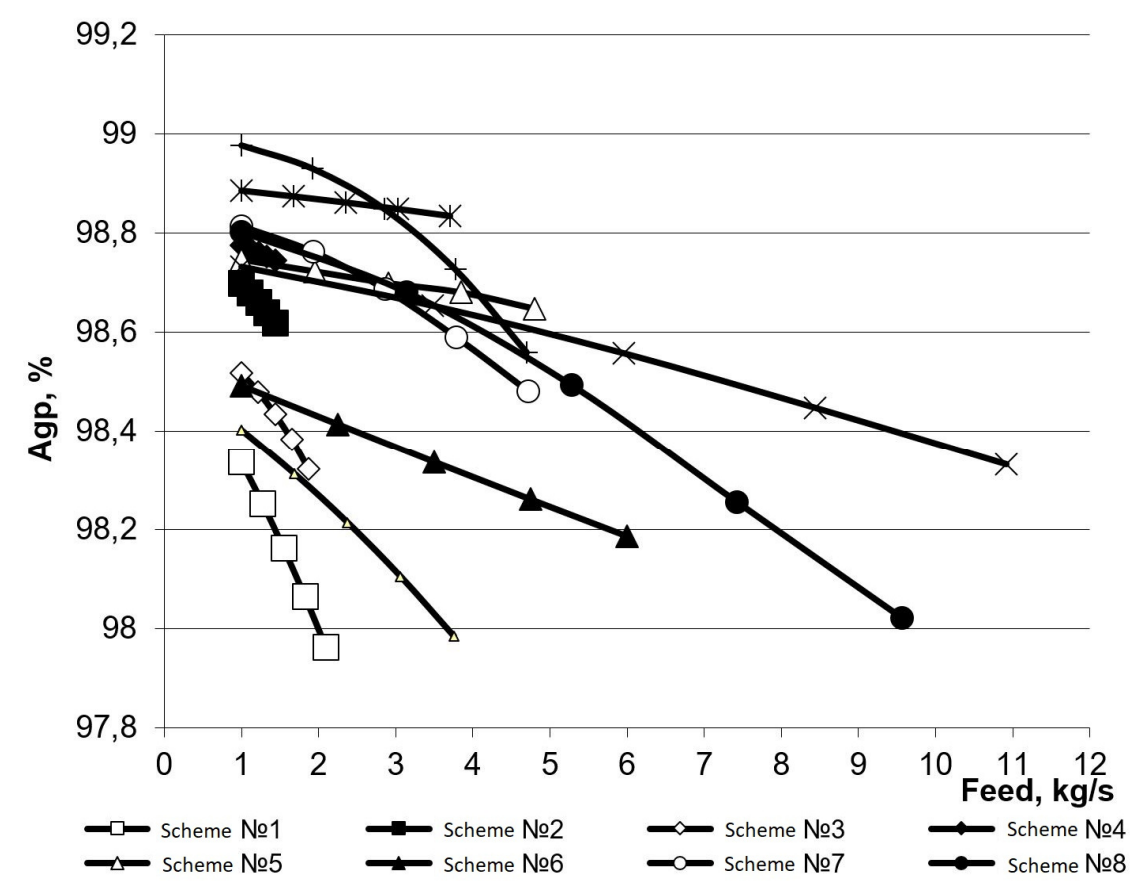

Fig.3. Clean grain material, cleaned by sieve modules with different functioning schemes.

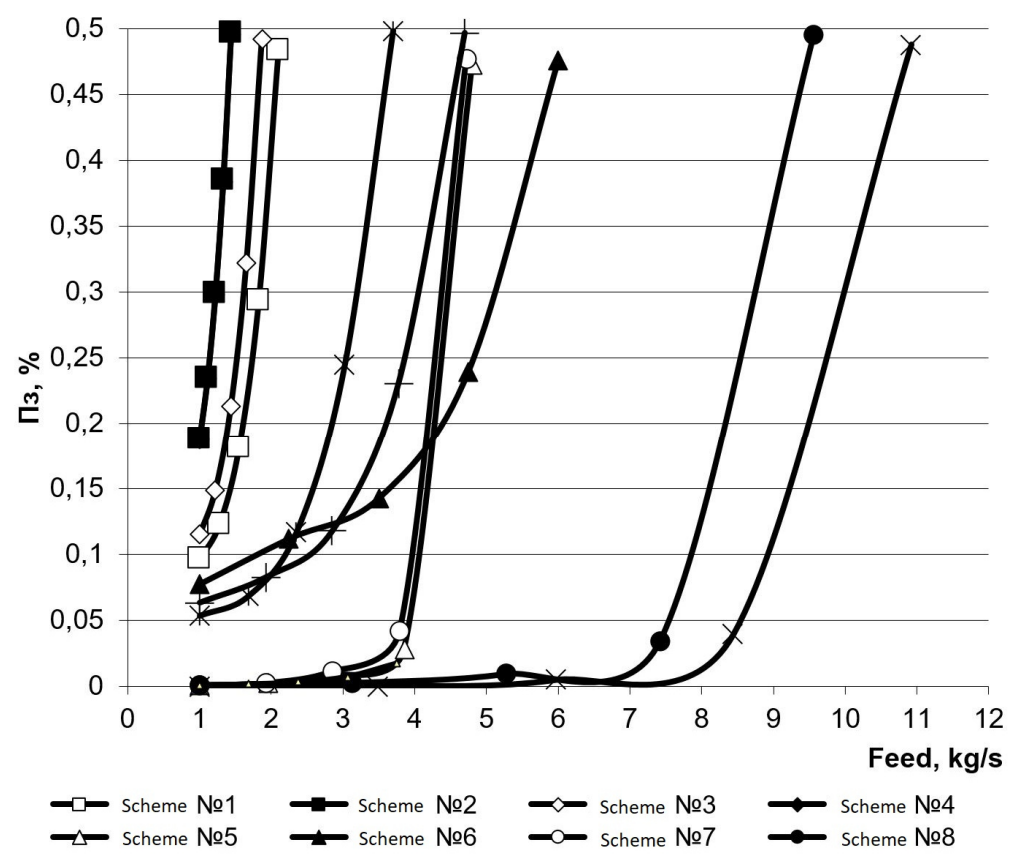

Fig. 4.Loss of grain material during cleaning by sieve moduleswith different functioning schemes. 


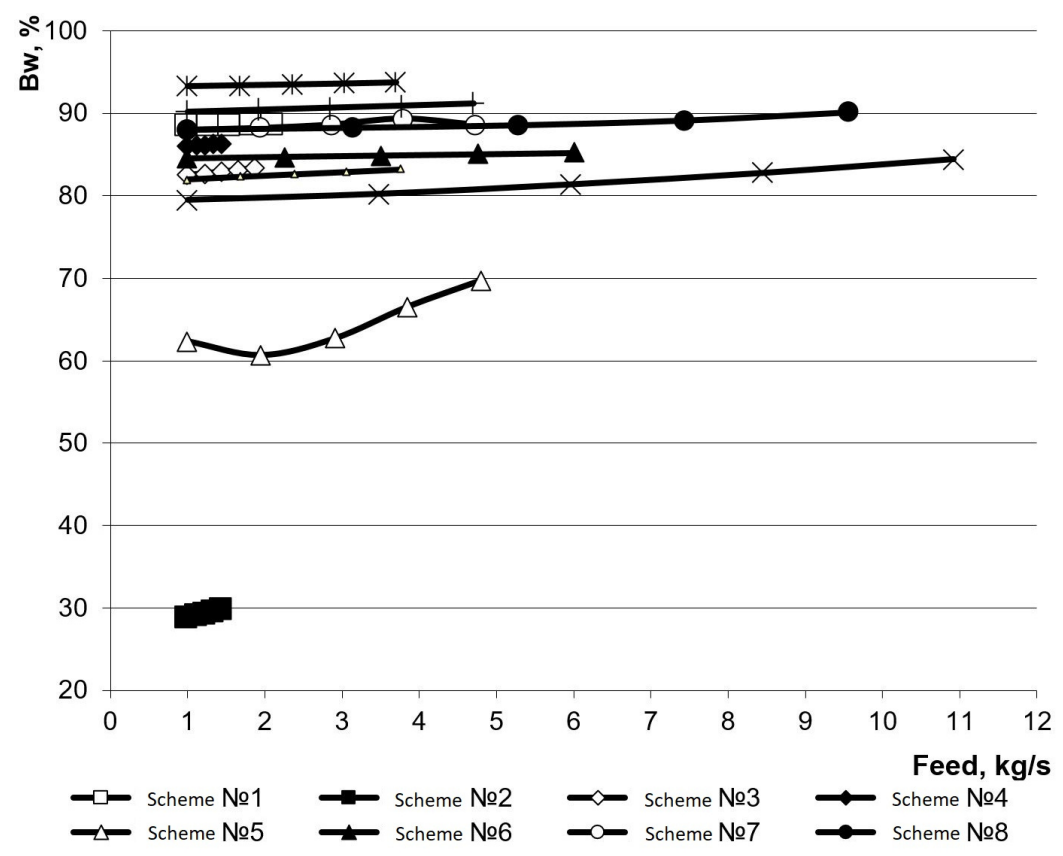

Fig. 5.The completeness of the separation of weed impurities during cleaning by sieve modules with different functioning schemes.

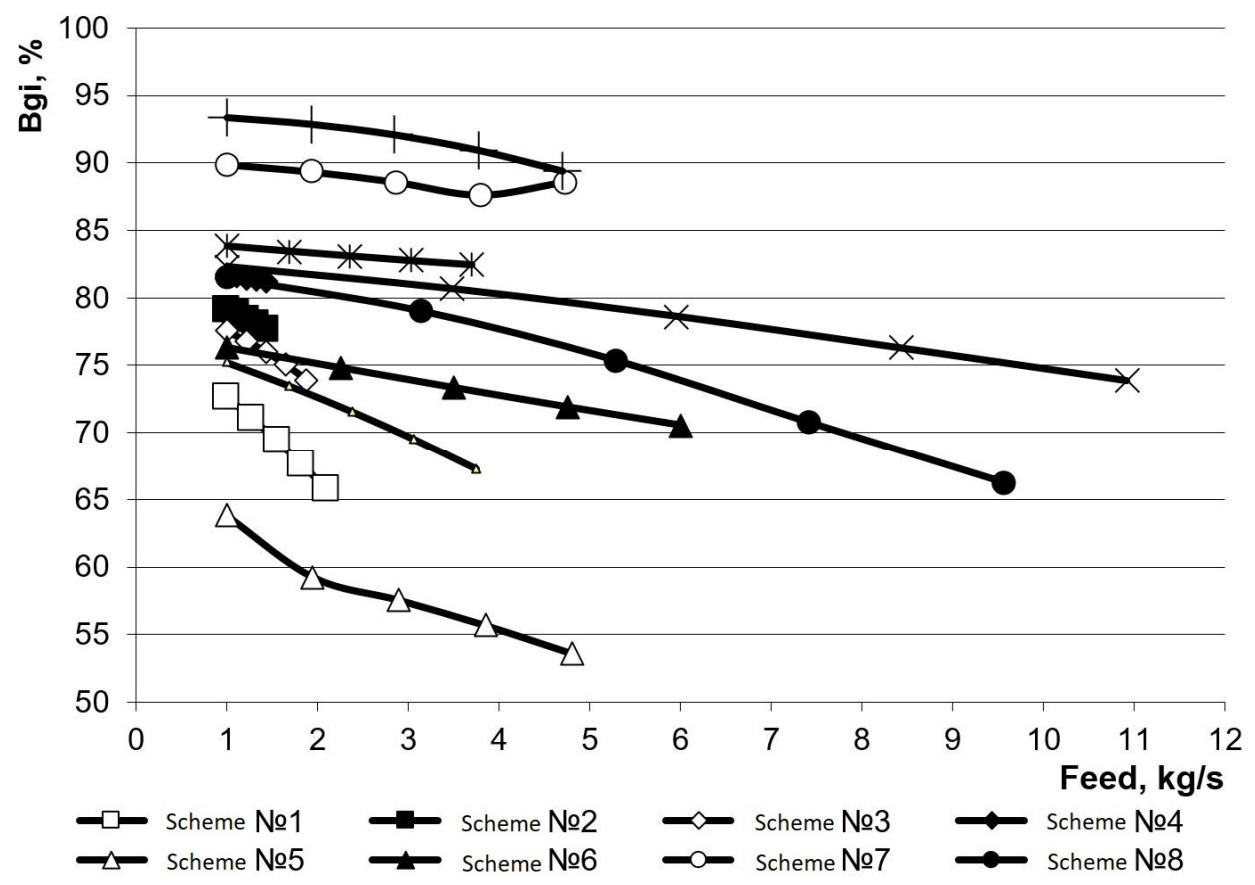

Fig. 6. The completeness of the separation of grain impurities during cleaning by sieve modules with different functioning schemes. 


\section{Conclusions}

The obtained dependences allow a comparative analysis of the functioning of the sieve modules for the grain cleaning machines. The productivity of machines is limited by a sharp increase in losses associated with the release of seed into the fraction of weed impurities. Schemes No. 8 and No. 10 showed the greatest productivity due to the developed sieve structure, however, it is necessary to further analyze the economic feasibility of these schemes, since the cost and subsequent operation of this system can show its economic inefficiency.

\section{References}

1. A. Giyevskiy, MEACS, 327, 042035 (2018) doi:10.1088/1757-899X/327/4/042035

P. Savinyh, Engineering for rural development, 17, 124-130 (2018)

2. Z. Dzhadyge, International journal of environmental \& science education, 11 (18), 11385-11392 (2016)

3. S. Bayshugulova, S. Seifullin, Kazakh Agrotechnical University Journal, 87(4), 74-82 (2015)

4. A. Linenk, Ulyanovsk State Agricultural Academy Journal, 2, 18 (2012)

5. Z. Jing, Journal of London University, 1, 17 (2013)

6. V. Saitov, Procedia Engineering, 150, 107-110 (2016) doi: 10.1016/j.proeng.2016.06.728

7. A. Butovchenko, MATEC, 224, 05012 (2018) doi: 10.1051/matecconf $/ 201822405012$

8. A. Doroshenko, MATEC, 224, 05013 (2018) doi: 10.1051/matecconf $/ 201822405013$

9. Y. Wang, Powder Technology, 346, 193-202 (2019) doi: 10.1016/j.powtec.2019.01.076

10. A. Butovchenko, Bull. DSTU, 3 (64), 16-21 (2012)

11. U. Ermoliev, Bull. DSTU, 7(68), 90-98 (2012) 